\title{
La comunicación interna en las cooperativas: la percepción de las personas trabajadoras sobre la escucha y la información
}

\author{
Onintza Belategi ${ }^{1}$, Mónica Gago ${ }^{2}$ y Txema Egaña ${ }^{3}$
}

Recibido: 12 de julio de 2018 / Aceptado: 31 de octubre de 2018

Resumen. El objetivo principal de este trabajo de investigación es conocer las percepciones y expectativas de las personas trabajadoras de una cooperativa del Grupo MONDRAGON sobre la comunicación interna. Se ha puesto especial interés en conocer: por un lado, las percepciones de las personas trabajadoras sobre la información transmitida por la cooperativa; por otro lado, sus percepciones sobre cómo realizan la escucha los directivos y mandos intermedios cuando les trasladan sus preocupaciones y expectativas. Asimismo, con la intención de conocer si existen diferencias, se han analizado las percepciones indicadas teniendo en cuenta las siguientes variables: ser miembro de algún órgano, tipo de mano de obra, situación laboral y sede. Para la realización de este estudio de caso se han combinado la metodología cualitativa y cuantitativa: se ha llevado a cabo una recogida de datos cuantitativos a través de un cuestionario propio y datos cualitativos a través de la realización de entrevistas semiestructuradas. Los resultados de la investigación sugieren que por lo general, aunque las personas trabajadoras de la cooperativa se sienten informadas, tienen la percepción de que se les escucha poco a la hora de trasladar sus preocupaciones y expectativas; al mismo tiempo, encontramos diferencias estadísticas significativas al analizar esos mismos datos por variables.

Palabras clave: Comunicación Organizacional; Comunicación Interna; Comunicación; Cooperativa; Grupo Mondragon.

Claves Econlit: M14; J54; P13.

\section{[en] Internal communication in cooperatives: the perception of working people about listening and information}

\begin{abstract}
The main objective of this research is to discover the perceptions of internal communication of the work force of a cooperative company, member of the MONDRAGON Group. Our focus was two-fold, first, on exploring workers' perceptions of the information transmitted to them by the cooperative; and second, on their perceptions of how well managers and supervisors listen to frontline workers' expressions of concerns and expectations. Furthermore, in order to identify possible specific relationship between communication and other key factors, the study controls for the following variables: governance/management body membership, type of workforce (white collar and blue collar), type of contract (member or employee), and place of work. To carry out this case study,
\end{abstract}

1 Universidad de Mondragón, España

Dirección de correo electrónico: obelategi@mondragon.edu

2 Universidad de Mondragón, España

Dirección de correo electrónico: mgago@mondragon.edu

3 Universidad de Mondragón, España

Dirección de correo electrónico: tegana@mondragon.edu 
the authors used both qualitative and quantitative methods. Quantitative data were collected through a questionnaire, and qualitative data through semi-structured interviews. The results of the research suggest that, in general, the workers of the cooperative feel informed, but they perceive that their concerns and expectation are scarcely heard. We also examine interesting differences uncovered by holding constant the specific variables mentioned.

Keywords: Organizational Communication; Internal Communication; Communication; Cooperative; Mondragon Group.

Sumario. 1. Introducción. 2. Marco teórico. 3. La responsabilidad de MONDRAGON con la comunicación interna. 4. Objetivos de la investigación. 5. Metodología. 6. Resultados. 7. Discusión de los resultados. 8. Conclusiones, límites de la investigación y futuras líneas de actuación. 9. Referencias bibliográficas.

\section{Introducción}

En la literatura sobre gestión empresarial se ha revitalizado la apuesta por la comunicación interna como herramienta estratégica. De hecho, varias investigaciones apuntan a la relación existente entre una adecuada política de comunicación interna y distintos ámbitos relacionados con la empresa, como por ejemplo: la satisfacción de las personas trabajadoras (Bustamante, 2013), su motivación (Bustamante, 2013; Opitz y Hinner, 2003), la identificación de las mismas con la empresa (Smidts, Pruyn y Van Riel, 2001), su productividad (Holá, 2012; Clampitt y Downs, 1993) y los resultados de la empresa (Nikolić, Terek, Vukonjanski e Ivin, 2012).

En el día a día de trabajo la persona trabajadora demanda información, quiere saber qué está pasando en su entorno de trabajo, cuáles son las novedades de la empresa, sus logros o las decisiones tomadas; esto es, necesita estar informada (Vilanova, 2013; Ruck y Welch, 2012). Por ello, durante años se han ido creando estrategias de comunicación interna para difundir la información sobre la empresa entre las personas trabajadoras. No obstante, todos los esfuerzos de las organizaciones no deben centrarse en informar. Hay que concebir la comunicación interna de manera más amplia pues la práctica eficaz de la comunicación interna implica, además de informar, escuchar. De hecho, Varona (2007) recuerda que una de las necesidades más importantes del ser humano es la de ser escuchado y, paradójicamente, es una de las más difíciles de satisfacer. Por añadidura, uno de los retos que debe plantearse una corporación empresarial para gestionar correctamente su comunicación interna es el de practicar la escucha activa, y ello se consigue, entre otros aspectos, atendiendo a las personas que en ella trabajan (Tessi, 2012). Si la escucha es importante en la gestión empresarial, aún lo es más dentro de las sociedades cooperativas, donde la persona trabajadora tiene, en muchos casos, el doble rol de trabajadora-propietaria.

Es por ello precisamente que el objetivo de este artículo es conocer las percepciones de las personas trabajadoras sobre la comunicación interna de una cooperativa del Grupo MONDRAGON. Se ha prestado especial interés en conocer no solo las percepciones de las personas trabajadoras sobre la información transmitida por la cooperativa, sino también sus percepciones sobre cómo realizan la escucha los directivos y mandos intermedios de la cooperativa cuando las personas trabajadoras les trasladan sus preocupaciones y expectativas. A su vez, 
queremos resaltar la importancia de realizar la investigación en una cooperativa, y más aún en una cooperativa del Grupo MONDRAGON. Primero, porque las cooperativas son empresas que tratan de integrar otro tipo de valores a la lógica económica -la centralidad de la persona, la práctica de la democracia y la autoorganización, la solidaridad e inter-cooperación, el enraizamiento y el compromiso social, entre otros-, y la comunicación cumple un papel fundamental en este intento de caminar hacia modelos más humanos de hacer empresa y economía que representan las cooperativas. Segundo, porque no hay ninguna investigación sobre MONDRAGON que abarque temas relacionados con la comunicación interna, ya que normalmente las investigaciones se han enfocado a estudios sobre organización democrática, participación, valores y decisiones adoptadas por las cooperativas del grupo ante la globalización. Todo ello sin olvidar que MONDRAGON es un referente a nivel mundial en el ámbito de la economía social. Precisamente, MONDRAGON ocupa el primer puesto en el ranking mundial de cooperativas de industria (TU Lankide, 2014). Constituye, además, el primer grupo empresarial del País Vasco y el décimo en el ranking de las principales empresas españolas, con implantaciones comerciales y productivas en los cinco continentes (MONDRAGON, 2018).

El artículo se ha organizado de la siguiente manera: primero, en el marco teórico, se presenta un breve repaso de la literatura para conocer la importancia de la transmisión de información dentro de la empresa, así como de la importancia de la escucha. A continuación, se exponen los objetivos de la investigación y se describe la metodología llevada a cabo para dar respuesta a los objetivos planteados, incidiendo en su especificidad, diseño y procedimiento. Acto seguido se muestran los resultados obtenidos en la investigación, tanto los cuantitativos como los de corte cualitativo. Finalmente, se da paso a la discusión de resultados, las conclusiones, los límites de la investigación y las futuras líneas de actuación.

\section{Marco teórico}

En el ámbito de la comunicación corporativa son varios los autores (Moreno, Crístina y Pet, 2012; Cornella, 2002; Catalá, 2001, Timmerman, 2000) que indican que la información es un recurso estratégico básico, uno de los más importantes de las organizaciones, ya que es el elemento de cohesión mediante el cual se logran los objetivos de la empresa. Por tanto, resulta indispensable para planificar y tomar decisiones. En ese mismo sentido se pronuncia también Gómez Aguilar (2007) que concluye que las personas trabajadoras que forman parte de los flujos de información son además de más productivas, más participativas y tienen mayor seguridad a la hora de tomar decisiones. Siendo eso así, no es de extrañar que en las últimas décadas se hayan realizado infinidad de investigaciones para saber qué tipo de información (y en qué cantidad) reciben las personas trabajadoras, y qué herramientas utilizan para recibir dicha información; se pretende conocer asimismo si la información recibida es comprensible y qué factores -como puede ser el clima laboral- pueden influir su calidad.

De hecho, las organizaciones saben que el primer agente en el que hay que pensar para lograr metas es el equipo humano que sustenta su negocio (Rosenbluth, 
McFerrin, 2004). Así pues, debemos de ser conscientes de la importancia de mantener informadas a las personas trabajadoras (Bustamante, 2013b).

Sin embargo, D’Aprix (1999) y Bartoli (1992) recuerdan que, además de informar a las personas trabajadoras, resulta fundamental conocer la opinión de las mismas en relación a los flujos de información. Las prácticas de vanguardia demuestran que la comunicación en el ámbito laboral alcanza logros profundos cuando se gestiona de manera transversal con estrategias que conciben a los distintos niveles de la organización como emisores de mensajes y no sólo como receptores de información. Es decir, las empresas, preocupadas habitualmente por la incidencia de los mensajes en las personas de su organización, van entendiendo que la escucha resulta fundamental en la comunicación interna (Tessi, 2012). Si bien es importante que la organización dé a conocer noticias de la misma, es también importante darse cuenta de que esta es solo una de las funciónes de la comunicación interna. Dejar que toda la estrategia de comunicación interna sea acaparada por la transmisión de mensajes informativos sobre la empresa es un error frecuente. Tessi (2012:65) enfatiza la idea de que para mejorar la comunicación interna de cualquier empresa lo primero que hay que hacer es escuchar a los trabajadores: "Una de las decisiones más inteligentes y estratégicas que puede tomarse en materia de comunicación interna es, justamente, priorizar las acciones de escucha por encima de cualquier cosa".

Así pues, el saber escuchar es uno de los pilares de la comunicación corporativa efectiva. Entre las principales acciones que puede realizar una persona o directiva para comunicarse con efectividad, escuchar es probablemente la más importante (Tessi, 2012). Precisamente, los mejores comunicadores destacan por su capacidad de escucha (Vilanova, 2013). En términos organizacionales la situación no es diferente. Las instituciones mejor comunicadas internamente son aquellas que tienen gran sensibilidad en esta materia y lo demuestran destinando una parte importante de sus inversiones comunicacionales para escuchar a sus colaboradores (Tessi, 2012).

El no saber escuchar influye negativamente en la productividad de la empresa (Tessi, 2012), en su efectividad y su clima comunicacional (Hunt, Cusella, 1983), así como en su rentabilidad, en sus ventas, en su reputación, en el servicio ofertado a los clientes (Steil, Bommelje, 2004) y en la satisfacción de las personas trabajadoras (Varona, 2007), ya que su insatisfacción afecta a la calidad del trabajo y a la entrega o compromiso con la organización. Por lo tanto, pudiera parecer que el escuchar bien o no, puede ser la diferencia entre el éxito o el fracaso tanto para las personas trabajadoras como para la empresa. De hecho, las personas trabajadoras se sienten más valoradas cuando la Dirección se muestra dispuesta a escuchar (Mishra, 2007): cuanto más escuchadas, respetadas y consultadas se sienten las personas trabajadoras, más se implican en la resolución de posibles conflictos (Simard, Marchand, Brossard, 1990).

A pesar de todos los beneficios de la escucha, y aunque hace ya más de medio siglo Zelko (1954) destacó que para un adecuado proceso comunicativo era fundamental esta práctica, un par de años después Teplow (1956) recordaba que no se estaba realizando un buen trabajo a la hora de escuchar a las personas trabajadoras. Años más tarde, algunas investigaciones seguían indicando que las personas trabajadoras se sentían poco escuchadas (Dircom, 2002; Inforpress, 
2002). En ese mismo sentido, Zerfass, Verčič, Verhoeven, Moreno y Tench (2015) indican que mientras que la mayor parte de los gestores han establecido estrategias para que los mensajes de la organización lleguen a los diferentes grupos de interés clave $(78,3 \%)$, solo la mitad de ellos ha integrado sistemas de escucha activa para monitorizar la opinión de los mismos en la toma de decisiones estratégicas. Es por todo ello por lo que Gonzalo (2012:64) recuerda que debemos reflexionar sobre el tiempo que dedicamos a escuchar: "Todos los que nos dedicamos a la comunicación interna deberíamos preguntarnos con frecuencia cuánto tiempo y cuántas energías y recursos dedicamos a hablar/escribir/emitir y cuánto a escuchar, tomar el pulso y comprender a nuestros públicos internos". Igualmente, Macnamara (2015) y Varona (2007) indican que la escucha es un acto olvidado o descuidado (Zerfass et al., 2015) en el mundo laboral, que son contados los superiores que son conscientes de ello y que hacen del escuchar una de sus responsabilidades más importantes (Varona, 2007). A su vez, la investigación realizada por Inforpress (2002) concluyó que las personas trabajadoras de las empresas de gran facturación de España tienen la impresión de que se les escucha poco. En la misma época, en la investigación realizada por Dircom (2002) se preguntó a los mandos si se sentían escuchados y apoyados por su dirección superior cuando les trasladaban las preocupaciones y expectativas de sus colaboradores. Los resultados, sin ser desalentadores, indicaron que queda espacio para la mejora: un 33\% de esos mandos se sienten "algo escuchados" y más del 5\% afirma que su "dirección superior no dedica tiempo a esos temas". En el caso de los trabajadores, por el contrario, el 17\% afirma que los mandos intermedios no conocen sus preocupaciones y expectativas. Por su parte, Truss, Soane, Edwards, Wisdom, Croll y Burnett (2006) realizaron un estudio en el que además de centrar el foco de atención en la escucha, analizaron la comunicación teniendo en cuenta diferentes variables. En el mismo se concluyó que la percepción del sentimiento de escucha varía en función del nivel de estudios, del tipo de contrato y de los diferentes cargos de los trabajadores. Concretamente, concluyeron que los graduados, los trabajadores con contrato flexible y las personas encargadas de supervisar el trabajo de los demás se sienten más escuchados que las personas con un bajo nivel de estudios, los trabajadores que no tienen un contrato laboral flexible y las personas que no tienen la labor de supervisar el trabajo de los demás.

Cabe destacar que, según Macnamara (2015), en las empresas tanto públicas como privadas no se ha prestado suficiente atención a la escucha. En cuanto a las cooperativas se refiere, hasta la fecha no se ha realizado ningún estudio en las cooperativas, en general, ni en el Grupo MONDRAGON, en particular, para conocer la percepción que tienen las personas trabajadoras sobre cómo realizan la escucha los directivos y mandos intermedios, aunque la centralidad de la persona y la práctica de la democracia adquieren un papel importante en ellas.

Ahora bien, como la experiencia cooperativa de MONDRAGON ha recibido una gran atención por parte del mundo académico (Heras-Saizarbitoria, 2014), la comunicación interna ha sido analizada de forma indirecta por algunos autores que han investigado sobre los valores, la participación, la gobernanza o la internalización en MONDRAGON, temas que guardan una estrecha relación con la comunicación. Así, por ejemplo, la investigación realizada por Bretos, Errasti y Marcuello (2018) indica que la presencia de las cooperativas de MONDRAGON 
en el exterior intensifica la tensión entre la eficiencia económica y la participación de las personas trabajadoras, quedando relegada la voz de estas últimas. Es por ello que la participación en la gestión debe ser una dimensión central en el modelo de empresas filiales de las cooperativas (Altuna, 2008). No obstante, el estudio realizado por Ortega (2011) concluyó que los órganos no están configurados para la participación activa de los socios, donde la mayor parte del tiempo se utiliza para la exposición de la información y el efecto que produce esta práctica es que no resulta fácil intervenir. En consonancia con lo mencionado, Heras-Saizarbitoria (2014) apuntó que es limitada la participación de las personas trabajadoras en los órganos de la cooperativa. Por su parte, Ortega y Uriarte (2015) concluyeron que una mayor dimensión de las cooperativas dificulta la gestión coherente en base a modos de gestión y principios cooperativos ya que se pierde la capacidad de comprensión por parte de los socios, resultando más complicado llegar con cierta rigurosidad hasta ellos. En esa misma línea, Errasti, Bretos y Nunez (2017) apuntan que un problema clave derivado de la dimensión de las cooperativas es el de las deficiencias existentes en el flujo de información entre las personas trabajadoras y la dirección.

Puesto que la comunicación interna debe empezar por tomar el pulso, por conocer, por observar y desentrañar cuál es la situación de las personas de la compañía, qué sienten, qué piensan y qué esperan (Vilanova, 2013), mediante esta investigación se ha querido conocer si se sienten informadas y escuchadas las personas trabajadoras de una cooperativa del Grupo MONDRAGON. Es decir, si se sienten informadas y escuchadas las personas trabajadoras de las empresas que reivindican, entre otros aspectos, el compromiso social y la organización democrática. Asimismo, y con el objetivo de conocer si existen diferencias en los datos obtenidos en este trabajo de investigación se han analizado los datos indicados teniendo en cuenta las siguientes variables: ser miembro de órganos, tipo de mano de obra, situación laboral y sede. Con respecto a la variable ser miembro de órganos, Thompson (2000) indicó que la jerarquía de las personas trabajadoras influye en la percepción del sentimiento de información. De manera análoga, Lingán (2003) concluyó que cuanto menor es el cargo de las personas trabajadoras, menor es la percepción del sentimiento de información de las mismas. Por otro lado, Truss y su equipo (2006) apuntaron que la percepción del sentimiento de información es mayor en las personas con estudios superiores. Igualmente, tal y como se ha señalado anteriormente, Truss y su equipo (2006) destacaron que los graduados, las personas trabajadoras con contrato flexible y las personas encargadas de supervisar el trabajo de los demás se sienten más escuchadas. Con respecto al efecto sede, Bustamante (2013b) concluyó que la percepción de las personas sobre la información recibida varía en función de la sede de trabajo. Asimismo, Goldhaber y Porter, Yastes y Lesniak (1978) indicaron que a mayor distancia entre la fuente de información y el receptor disminuye la cantidad y la posibilidad de pedir información, empeora la calidad de la misma y aumentan los rumores. Más allá de la justificación académica, también el presidente de la cooperativa objeto de estudio estimó interesante y necesario poder analizar los resultados a través de las variables indicadas. 


\section{La responsabilidad de MONDRAGON con la comunicación interna}

En las cooperativas se comparten algunas potencialidades claves como la centralidad de la persona y la práctica de la democracia y la participación en la gestión, aspectos que ponen de manifiesto la necesidad de una adecuada política de comunicación. Precisamente, en el primer trimestre del 2018 se ha estrenado el Foro de Comunicación de MONDRAGON, un evento que pretende crear un espacio para compartir experiencias y al mismo tiempo poner en valor el carácter estratégico de la comunicación en las cooperativas (TU Lankide, 2018).

Desde sus inicios, el Grupo MONDRAGON se ha ido responsabilizando de la comunicación. Los principios cooperativos son una serie de lineamientos generales por los que se rigen las cooperativas y constituyen la base filosófica del movimiento cooperativo. Son diez -libre adhesión, organización democrática, soberanía del trabajo, carácter instrumental y subordinado del capital, participación en la gestión, solidaridad retributiva, inter-cooperación, transformación social, carácter universal y educación- y se aprobaron en el primer Congreso Cooperativo celebrado en octubre de 1987. Dos de los diez principios mencionados, más concretamente el segundo y el quinto, recogen la importancia de la comunicación interna:

- Organización democrática: este principio reivindica la igualdad básica de los socios trabajadores en lo que respecta a sus derechos a ser, poseer y conocer.

- Participación en la gestión: este principio exige el desarrollo progresivo de la autogestión y consecuentemente de la participación de los socios en el ámbito de la gestión empresarial, lo que, a su vez, requiere el desarrollo de los mecanismos de participación adecuados, transparencia informativa, consulta y negociación de las decisiones y aplicación de planes de formación y promoción interna.

Las políticas básicas sirven de enmarque estratégico ya que indican el rumbo a seguir de la Corporación, guiada siempre por las políticas socio-empresariales. Estas se aprueban en el Congreso y son especialmente importantes porque definen los objetivos de la Corporación y perfilan las líneas básicas de actuación para alcanzar el reto marcado. Es decir, señalan los objetivos de cada periodo (20012004, 2005-2008, 2009-2012, 2013-2016, 2017-2020) y las cooperativas del grupo tienen que trasladarlas a sus respectivos negocios mediante el Plan de Gestión.

Hasta el periodo 2005-2008, a la comunicación en MONDRAGON se le daba un enfoque más bien tecnológico. Sin embargo, el periodo 2009-2012 aparece como un punto de inflexión, pues a partir de ese momento cambia considerablemente la forma de entenderla. Se concibe la comunicación, y sobre todo la comunicación interna, como algo más que simples herramientas y canales. Se relaciona la comunicación interna con el liderazgo y la participación, implicación, cohesión y motivación de las personas trabajadoras. De igual forma, aflora la importancia que tienen los líderes y sus actitudes en el adecuado ir y venir de los flujos de información, y también el saber escuchar, las emociones o el respeto. 
Teniendo en cuenta todo lo anterior, parece que la comunicación interna es estratégica para el Grupo MONDRAGON (así se recoge también en el acta del VIII. Congreso Cooperativo del 21 de mayo de 2003). Sin embargo, aunque parezca paradójico, apenas se ha investigado sobre la comunicación interna y sobre la habilidad de escucha de los mandos directivos en el grupo de cooperativas.

\section{Objetivos de la investigación}

El objetivo principal de este trabajo de investigación es conocer las percepciones de las personas trabajadoras sobre la comunicación interna de una cooperativa del Grupo MONDRAGON.

Para dar respuesta al objetivo mencionado se han diseñado las siguientes preguntas de investigación:

1. ¿Qué percepciones tienen las personas trabajadoras sobre la información transmitida por la cooperativa?

2. ¿Qué percepciones tienen las personas trabajadoras sobre cómo realizan la escucha los directivos y mandos intermedios de la cooperativa cuando les trasladan sus preocupaciones y expectativas?

Asimismo, se han analizado las percepciones indicadas teniendo en cuenta las siguientes variables: ser miembro de órganos, tipo de mano de obra, situación laboral y sede. Como órganos de la cooperativa se han incluido el Consejo de Dirección, el Consejo Rector y el Consejo Social. Dentro de mano de obra se distingue entre mano de obra directa e indirecta. En la variable situación laboral se diferencian personas socias y contratadas. Por último, en la variable sede se incluyen las opiniones de las personas trabajadoras de las tres sedes con las que cuenta la cooperativa en cuestión.

\section{Metodología}

El universo de estudio de esta investigación está formado por las personas trabajadoras de una cooperativa del Grupo Fagor $^{4}$, dentro de MONDRAGON, que cuenta con una trayectoria superior a 40 años en el sector de máquina-herramienta, en el que es uno de los líderes mundiales. La sede central está ubicada en ArrasateMondragón y tiene otras dos sedes en el País Vasco. Esta cooperativa cuenta con 404 personas trabajadoras, tanto de mano de obra directa como de mano de obra indirecta; más de la mitad son tituladas, con estudios medios y superiores.

Para la consecución de los objetivos indicados se ha adoptado un enfoque metodológico basado en el estudio de casos: primero, porque a través de esta metodología se mide y registra la conducta de las personas involucradas en el

4 Cuando se llevó a cabo la parte práctica - cuantitativa y cualitativa- de la investigación el Grupo Fagor estaba constituido por 9 cooperativas y unas 9.000 personas trabajadoras, más o menos. Sin embargo, desde noviembre de 2013, y tras el concurso de acreedores y posterior venta de Fagor Electrodomésticos S.Coop., el Grupo Fagor alberga 8 cooperativas -Copreci, Fagor Arrasate, Fagor Automation, Fagor Ederlan, Fagor Electrónica, Fagor Industrial, Mondragon Assembly y Fagor S.Coop.- y unas 7.200 personas trabajadoras. 
fenómeno estudiado, sin centrarse únicamente en la información obtenida a través de cuestionarios (Yin, 2009); segundo, porque este enfoque permite trabajar sobre un problema en particular, pero considerándolo en toda su complejidad (Stake, 1999); tercero, porque este modo de aproximación permite explorar de forma más profunda y obtener un conocimiento más amplio sobre cada fenómeno, lo cual permite la aparición de nuevas señales sobre los temas que emergen (Chetty, 1996); y por último porque el método de estudio de casos es adecuado para describir e interpretar los fenómenos de empresa (Castro, 2010). Los estudios de caso también han sido objeto de críticas por parte de algunos autores (entre otros: Díaz de Salas, Mendoza y Porras, 2011; Villareal y Landeta, 2010), especialmente porque lo han limitado al enfoque cualitativo. Es por ello que en la investigación se ha diseñado el estudio de casos tanto desde un enfoque cuantitativo -basado en cuestionarios- como desde uno cualitativo -basado en entrevistas-.

Una vez establecidos los objetivos de estudio, se llevó a cabo una amplia revisión bibliográfica para identificar herramientas que ayudasen a dar respuesta a los objetivos planteados. Aunque se encontraron algunas, estas daban una solución parcial a los objetivos planteados en el estudio y, por ello, se decidió crear un cuestionario propio basado en las propuestas de Downs (1988), Del Pozo (1995), Dircom (2002) y Belategi (2006). Para la confección del cuestionario se contó con la ayuda de Pablo Gonzalo, Federico Varona y George Cheney, expertos que cuentan con una dilatada trayectoria profesional en el ámbito de la comunicación corporativa y la comunicación interna y de investigadores del Departamento de Comunicación de Mondragon Unibertsitatea. Se analizó la validez de construcción, la de contenido y la de didáctica o permanente. De este modo, se ha asegurado que el cuestionario responde a los objetivos de la investigación (Pérez Serrano, 1998).

La cooperativa en cuestión tenía en el momento del estudio cuantitativo (año 2012) una población interna de 422 personas trabajadoras. El cuestionario se entregó en mano a todas las personas trabajadoras y se recogieron 224, por lo que la muestra de la investigación representa el 53,08\% de la población. Asimismo, el margen de error es de $+-4,49 \%$ para toda la muestra, mientras que el nivel de confianza es de $97,08 \%$ y $p=q=50 \%$.

Se realizaron entrevistas semiestructuradas siguiendo las indicaciones de Bisquerra (2009), Gómez (2006) y Flick (2007). Por un lado, porque se determina de antemano cuál es la información relevante que se quiere conseguir, por lo que el entrevistador dispone de un guion que recoge los temas que debe tratar a lo largo de la entrevista; por otro lado, porque se hacen preguntas abiertas dando oportunidad a recibir más matices de la respuesta, permitiendo entrelazar temas; y por último, porque es más probable que los sujetos entrevistados expresen sus puntos de vista en las entrevistas semiestructuradas que en una entrevista estandarizada o en un cuestionario.

Se llevaron a cabo ocho entrevistas semiestructuradas: dos a miembros del Consejo Rector, otras dos a miembros del Consejo Social y cuatro a personas trabajadoras que no son miembros de ningún órgano. Estas últimas cuatro entrevistas se dividen, a su vez, entre personas trabajadoras de mano de obra directa e indirecta -se realizaron dos entrevistas a cada tipo de mano de obra-. Se decidió entrevistar a estas personas ya que en los resultados del cuestionario los 
datos más significativos se encontraron en las variables ser miembro de órganos y tipo de mano de obra. Las entrevistas se desarrollaron en el año 2013.

Las preguntas tanto del cuestionario como de las entrevistas están diseñadas para conocer las percepciones de las personas trabajadoras sobre la comunicación interna de la cooperativa objeto de estudio. Por consiguiente, los ítems utilizados indagan, entre otros, en la percepción de las personas trabajadoras sobre la información trasmitida por la propia cooperativa, en el tipo de información que transmite y las herramientas y canales utilizados para ello, en la percepción de las personas trabajadoras sobre cómo realizan la escucha los directivos y mandos intermedios, y, finalmente, en las herramientas y canales de los que dispone la cooperativa para que la opinión de las personas trabajadoras sea conocida por la Dirección (las encuestas de opinión, el plan de comunicación interna y las acciones de comunicación interna).

Por último, y antes de presentar los resultados, queremos destacar que el concurso de acreedores de Fagor Electrodomésticos (tal y como se indica en el pie de página $n^{\circ} 4$, Fagor Electrodomésticos es una cooperativa autónoma diferente, pero del mismo grupo que la cooperativa de objeto de estudio) nos hizo reflexionar, forzosamente, sobre la investigación que estábamos llevando a cabo. Desde el primer momento en el que tuvimos conocimiento del concurso de acreedores fuimos muy cautos para conocer si los acontecimientos podían influir en la investigación. Para dar respuesta a dicha corazonada, se decidió volver a entrevistar a las ocho personas participantes en el trabajo de campo cualitativo, tan solo veinte días después de que Fagor Electrodomésticos entrara en concurso de acreedores. Más información sobre esta cooperativa en concreto y su crisis se puede encontrar en Arando y Arenaza (2018), Errasti, Bretos y Nunez (2017) y Ortega y Uriarte (2015). Todas las personas entrevistadas indicaron que si las entrevistas realizadas en mayo hubieran sido realizadas en diciembre -cuando Fagor Electrodomésticos entró en concurso de acreedores-, sus respuestas hubieran sido las mismas. Por consiguiente, las entrevistas de diciembre de 2013 apuntan a que los datos obtenidos de la investigación empírica son válidos. Es decir, que el concurso de acreedores de Fagor Electrodomésticos no influyó en los resultados de la investigación.

\section{Resultados}

En este apartado se presentarán los datos cuantitativos y cualitativos teniendo en cuenta las preguntas de investigación: (1) ¿Qué percepciones tienen las personas trabajadoras sobre la información transmitida por la cooperativa?; (2) ¿Qué percepciones tienen las personas trabajadoras sobre cómo realizan la escucha los directivos y mandos intermedios de la cooperativa cuando les trasladan sus preocupaciones y expectativas? Todo ello se hará exponiendo en primer lugar el resultado general obtenido de los 224 cuestionarios y ocho entrevistas y, seguidamente, exponiendo los resultados teniendo en cuenta las variables -ser miembro de órganos, tipo de mano de obra, situación laboral y sede-. Así pues, podemos realizar comparaciones entre los distintos grupos de cada variable. Además, se han realizado contrastes de medias entre las variables. Para ello, se han 
utilizado las pruebas no paramétricas de Kruskal Wallis y Mann-Whitney, y las pruebas paramétricas ANOVA y $\mathrm{t}$ de Student. Las primeras, Kruskal Wallis y ANOVA, se han utilizado para realizar contrastes de diferencias de medias entre más de dos grupos; las segundas, Mann-Whiney y t de Student, para realizar contrastes de diferencias de medias entre dos grupos. Se decide aplicar ambas técnicas con el objetivo de comprobar la robustez de los resultados.

La variable ser miembro de órganos tienen un tamaño reducido en cada una de sus categorías: el Consejo de Dirección, el Consejo Rector y el Consejo Social. Los investigadores son conscientes de que los resultados de una muestra tan pequeña deben de ser analizados con cautela. Sin embargo, se estima interesante realizar contrastes entre las personas trabajadoras de los distintos órganos. Por consiguiente, y para garantizar la fiabilidad de los resultados obtenidos, se llevan a cabo diferentes acciones: por un lado, en las pruebas realizadas con las personas trabajadoras de los distintos órganos se certifica que los resultados se mantienen con respecto a las realizadas teniendo en cuenta la suma de los componentes de Consejo de Dirección, del Consejo Rector y del Consejo Social; por otro lado, se han aplicado las correcciones de Bonferroni.

Entre las personas trabajadoras que han respondido el cuestionario con respecto a la percepción de sentirse informados, el resultado medio ha sido de 6,17 (escala 1-10). En cuanto a la percepción de sentirse escuchados cuando les trasladan sus preocupaciones y expectativas a los directivos y mandos intermedios, el resultado medio ha sido de 5,65 (escala 1-10).

Asimismo, aunque las entrevistas sugieren que la cooperativa se preocupa por mantener informadas a las personas trabajadoras, queda latente que no hay unanimidad a la hora de afirmar que la cooperativa se preocupa por conocer las preocupaciones y expectativas de los trabajadores. La totalidad de las personas entrevistadas indican que ellas mismas se sienten informadas de los objetivos, metas, resultados y demás información concerniente a la cooperativa. No obstante, algunos entrevistados indican que aunque las personas trabajadoras disponen de canales para trasladar su opinión, desde esos canales no se realizan esfuerzos suficientes para escuchar a los trabajadores: "Se dispone de canales para conocer las percepciones y expectativas de los trabajadores, eso sí, la cooperativa no se esfuerza lo suficiente para recogerlos. Creo que la cooperativa debería de hacer algo más en ese sentido". En esa misma línea, un miembro del Consejo Social añade que tanto los mandos intermedios como la alta dirección no están muy predispuestos a escuchar, pues ante cualquier queja o preocupación se ponen a la defensiva utilizando frases como: "Lo que dices no es verdad, nosotros hacemos las cosas bien". Aun así, la totalidad de los entrevistados afirman que se sienten escuchados por sus superiores cuando les trasladan sus preocupaciones y expectativas.

A continuación, se van a desagregar los resultados por grupo de variable. En primer lugar, se presentan los resultados desagregados por la variable ser miembro de órganos: 
Tabla. 1. Promedios de la percepción del sentimiento de información y de escucha de los miembros del Consejo de Dirección (CD), Consejo Rector (CR), Consejo Social (CS) y las personas trabajadoras que no son miembros de órganos (OTROS) y contrastes de igualdad de medias entre los distintos grupos.

\begin{tabular}{l|l|l|l|l|l|l} 
& $\mathrm{CD}$ & $\mathrm{CR}$ & $\mathrm{CS}$ & OTROS & $\mathrm{K}-\mathrm{W}$ & ANOVA \\
\hline Información & 9,6 & 8 & 7,7 & 5,95 & $\mathrm{X}^{2}=34,76^{* * *}$ & $\mathrm{~F}=15,2^{* * *}$ \\
\hline Escucha & 8,8 & 4,28 & 4,9 & 5,15 & $\mathrm{X}^{2}=9,67 * *$ & $\mathrm{~F}=3,13 * *$ \\
\hline $\mathrm{N}$ & 5 & 7 & 10 & 202 & & \\
\hline
\end{tabular}

Nota: Escala 1-10. ***: significativo al 1\%; ** significativo al 5\%; * significativo al $10 \%$. NS: no significativo; KW: resultados del test no paramétrico de Kruskal-Wallis de igualdad de medias entre más de dos grupos; ANOVA: resultados del test paramétrico F de igualdad de medias entre más de dos grupos.

Los datos de la tabla indican que los promedios del sentimiento de información de los miembros de órganos son más altos que los de las personas trabajadoras que no son miembros de órganos. Sin embargo, no ocurre lo mismo con el promedio del sentimiento de escucha. En este último supuesto el promedio de los miembros del Consejo de Dirección es muy superior al del Consejo Rector, al del Consejo Social y al de las personas trabajadoras que no son miembros de órganos, destacando el bajo promedio (por debajo del aprobado) de los miembros del Consejo Rector y Consejo Social.

Para saber si la variable ser miembro de órganos influye en la percepción de sentirse informado y escuchado, se han utilizado la prueba no paramétrica de Kruskal-Wallis $\left(\mathrm{X}^{2}\right)$ y la prueba paramétrica de ANOVA. Los resultados obtenidos indican que la pertenencia a distintos órganos influye tanto en la percepción del sentimiento de información como en la percepción del sentimiento de escucha.

Además, para determinar entre que órganos hay diferencias significativas, se ha realizado el contraste no paramétrico de diferencias de medias de Mann-Whitney entre dos grupos (teniendo en cuenta las correcciones de Bonferroni) y el contraste paramétrico t de Student. A continuación, se presentan las comparativas dos a dos para los distintos órganos en ambos test. 
Tabla. 2. Contrastes de igualdad de medias para las distintas comparativas entre órganos:

Consejo de Dirección (CD), Consejo Rector (CR), Consejo Social (CS) y personas trabajadoras que no son miembros de órganos (OTROS).

\begin{tabular}{l|l|l|l|l|l|l|l|} 
& & CD-CR & CD-CS & $\begin{array}{l}\text { CD- } \\
\text { OTROS }\end{array}$ & CR-CS & $\begin{array}{l}\text { CR- } \\
\text { OTROS }\end{array}$ & $\begin{array}{l}\text { CS- } \\
\text { OTROS }\end{array}$ \\
\hline \multirow{2}{*}{ Información } & $\mathrm{MW}$ & $2 * * *$ & $3 * * *$ & $8,5 * * *$ & $31,5 \mathbf{N S}$ & $189 * * *$ & $395,5 * * *$ \\
\cline { 2 - 8 } & $\mathrm{T}$ & $3,79 * * *$ & $3,20 * * *$ & $13,48 * * *$ & $\begin{array}{l}0,554 \\
\mathbf{N S}\end{array}$ & $6,22 * * *$ & $3,332 * * *$ \\
\hline \multirow{2}{*}{ Escucha } & $\mathrm{MW}$ & $3 * *$ & $4,5 * * *$ & $116,5 * * *$ & $30 \mathbf{N S}$ & $580 \mathbf{N S}$ & $921,5 \mathbf{N S}$ \\
\cline { 2 - 8 } & $\mathrm{T}$ & $3,41 * * *$ & $3,32 * * *$ & $5,93 * * *$ &,$- 45 \mathbf{N S}$ &,$- 81 \mathbf{N S}$ &., $28 \mathbf{~ N S}$ \\
\hline
\end{tabular}

Nota: ***: significativo al $1 \% ; * *$ significativo al $5 \% ; *$ significativo al $10 \%$. NS: no significativo; MW: resultados del test no paramétrico de Mann-Whitney de igualdad de medias entre dos grupos; T: resultados del test paramétrico t de Student de igualdad de medias entre dos grupos.

En cuanto a la percepción del sentimiento de información se refiere, los resultados indican que hay diferencias significativas entre todos los grupos excepto en la comparativa entre Consejo Rector y Consejo Social, que presentan unos resultados muy similares. En cuanto a la percepción del sentimiento de escucha, los resultados obtenidos indican que hay diferencias significativas entre el Consejo de Dirección y el resto de órganos y personas no pertenecientes a órganos. Es decir, el Consejo de Dirección parece tener una percepción acerca de la escucha diferente al resto de personas trabajadoras.

Aunque la totalidad de los entrevistados indican que se sienten informados de los objetivos, metas, resultados y demás información concerniente a la cooperativa, los miembros del Consejo Rector creen que las personas trabajadoras sienten que se les oculta información, que hay falta de transparencia y que desconfían de lo que dicen los superiores: "Percibes esa sensación de que parece que estás ocultando información, y ese hecho no se da". Parece que los miembros de órganos se sienten preocupados, pues a su parecer la cooperativa se esfuerza realmente en informar a las personas trabajadoras, salvo en algunas cuestiones que estiman que no se pueden comunicar: "Se informa de todo menos de aquello que es crítico". Asimismo, añaden que "es verdad que la misma información no puede tenerla todo el mundo". En este sentido, un miembro del Consejo Social puntualiza que suele dudar sobre la información recibida de ciertos temas concretos como, por ejemplo, el de las contrataciones: "Nunca sabes si la información que te dan es cierta o no".

Asimismo, todas las personas entrevistadas, a excepción de una, indican que ser miembro de órganos influye en la percepción del sentimiento de información. Aseguran que los miembros de órganos manejan más información que las personas trabajadoras que no son miembros de órganos: "Claro que sí, los miembros de órganos disponen de mucha más información".

Al igual que sucede con la percepción del sentimiento de información, aunque la totalidad de los entrevistados afirman que se sienten escuchados por sus superiores cuando les trasladan sus preocupaciones y expectativas, indican que el colectivo de personas trabajadoras, por lo general, no se siente escuchado. Un 
miembro del Consejo Rector indica que la percepción de no sentirse escuchados es compartida también por el colectivo del Consejo Rector. En su opinión, en esa percepción influye el participar o no en los foros en los que se mueve dirección: "Yo creo que esa sensación de escucha va directamente relacionada no con estar en el Consejo Rector, sino con en qué otros foros participas". Añade que asistir a ciertos foros ayuda a tener una visión global de la cooperativa y, en consecuencia, a tener "peso" en las decisiones a tomar. A su vez, reconoce que son pocos los miembros del Consejo Rector que se encuentran en esa situación. Es por ello que cree que la baja percepción del sentimiento de escucha del colectivo del Consejo Rector puede deberse a este hecho. Igualmente, un miembro del Consejo Social indica que, aunque se siente escuchado, no se siente libre de trasladar a sus compañeros la información concerniente a la cooperativa a su manera. En consecuencia, se siente "utilizado". Dice que el guion ya está escrito y que su único deber es transmitirlo; reconoce que así no hay forma de contar las cosas a la manera de cada uno, y en ese sentido añade: "Te sientes un altavoz de lo que quieren que diga".

Los miembros del Consejo Rector, por su parte, indican que la cooperativa no realiza esfuerzos suficientes para conocer las preocupaciones y expectativas de las personas trabajadoras: "La cooperativa no se preocupa activamente por conocer las percepciones de los trabajadores". Aun así, señalan que la cooperativa cuenta con vías suficientes para conocer dichas percepciones: "Las mismas vías que están establecidas para transmitir la información pueden utilizarse para recoger información".

A continuación se muestran los resultados desagregados por la variable tipo de mano de obra ${ }^{5}$ :

Tabla. 3. Promedios de la percepción del sentimiento de información y de escucha de las personas trabajadoras de mano de obra directa e indirecta y los contrastes de igualdad de medias entre los distintos grupos

\begin{tabular}{l|l|l|l|l|} 
& Mano de obra indirecta & Mano de obra directa & MW & $\mathrm{T}$ \\
\hline Información & 6,85 & 5,58 & $2948,5 * * *$ & $5,50 * * *$ \\
\hline Escucha & 5,96 & 4,35 & $3229 * * *$ & $4,34 * * *$ \\
\hline $\mathrm{N}$ & 103 & 97 & & \\
\hline
\end{tabular}

Nota: Escala 1-10. ***: significativo al 1\%; ** significativo al 5\%; * significativo al $10 \%$. NS: no significativo; MW: resultados del test no paramétrico de Mann-Whitney de igualdad de medias entre dos grupos; T: resultados del test paramétrico t de Student de igualdad de medias entre dos grupos.

El promedio del sentimiento de información y de escucha de las personas trabajadoras de mano de obra indirecta es superior al promedio de las personas trabajadoras de mano de obra directa.

5 Se debe aclarar que en el cuestionario no se pregunta directamente a las personas trabajadoras si son de mano de obra directa o indirecta. Sin embargo, a partir del cargo y puesto recogidos en las respuestas se han podido identificar a 200 personas en las categorías de mano de obra directa e indirecta. 
Para saber si la variable tipo de mano de obra influye en la percepción de sentirse informado y escuchado se ha utilizado la prueba no paramétrica de MannWhitney y la prueba paramétrica t de Student. Los resultados obtenidos en ambas pruebas indican que el tipo de mano de obra influye tanto en la percepción del sentimiento de información como en la percepción del sentimiento de escucha.

En cuanto a las entrevistas se refiere, las personas trabajadoras de mano de obra directa indican que, por lo general, su colectivo no se siente informado. En su opinión, ese sentimiento se debe tanto a la falta de autoestima como a la falta de atención: "Hay mucha gente que dice que no nos informan y es porque, a menudo, no prestamos atención a lo que nos dicen".

Asimismo, casi la totalidad de los entrevistados afirman que el colectivo de personas trabajadoras de la cooperativa, por lo general, no se siente escuchado, haciendo especial hincapié en el colectivo de mano de obra directa. Aunque, en ese sentido, las personas trabajadoras de mano de obra directa también admiten que su colectivo no es muy propenso a hablar en los distintos foros de los que dispone la cooperativa: "Nos sentimos poco escuchadas ¿pero hablamos? ¿Expresamos nuestras preocupaciones?". Una trabajadora de mano de obra directa señala que su colectivo no da a conocer sus preocupaciones y quejas por sus propios complejos: "Tenemos muchos complejos". Por consiguiente, se avergüenzan de hablar delante de las personas trabajadoras de mano de obra indirecta, pues creen que son superiores a ellos: "Es gente experta, algunos tienen hasta doctorados".

Del mismo modo, la gran mayoría de los entrevistados percibe que ser una persona trabajadora de mano de obra directa o indirecta puede influir en la percepción del sentimiento de información y de escucha. En cuanto a la percepción del sentimiento de información, los entrevistados indican que las personas trabajadoras de mano de obra indirecta tienen más reuniones y contacto directo con las fuentes de información: "Si tienes al lado la fuente de información es más fácil que te llueva algo". No obstante, en cuanto a la percepción del sentimiento de escucha se refiere, los miembros del Consejo Rector creen que la baja percepción se debe al nivel de estudios de las personas trabajadoras. En su opinión, una baja formación dificulta la comprensión de la información recibida, y, por ende, la realización de preguntas y observaciones. Un miembro del Consejo Social afirma que las personas trabajadoras de mano de obra directa sienten que no están ni tan informadas, ni tan escuchadas como las personas trabajadoras de mano de obra indirecta debido a la actitud defensiva de los jefes inmediatos: "Se comportan como muros ante las quejas de las personas trabajadoras de mano de obra directa". Por ello, el colectivo de mano de obra directa tiene la sensación de que las quejas no van más allá de los jefes inmediatos. Una persona trabajadora de mano de obra indirecta atribuye a la cantidad de información recibida la influencia de la percepción del sentimiento de escucha. Por último, una persona trabajadora de mano de obra directa afirma que, en su opinión, su colectivo tiene la sensación de que son inferiores al resto: "Se nos considera como un tornillo que no da valor añadido al producto y eso hace que nos sintamos asî".

En tercer lugar, se recogen los resultados según la variable situación laboral: 
Tabla. 4. Promedios de la percepción del sentimiento de información y de escucha de los socios y contratados y los contrastes de igualdad de medias entre los distintos grupos

\begin{tabular}{l|l|l|l|l|} 
& Socio & Contratado & MW & T \\
\hline Información & 6,19 & 6 & 2258,5 NS & $0,53 \mathbf{~ N S}$ \\
\hline Escucha & 5,05 & 6,36 & $1822^{* *}$ & $-2,38^{* *}$ \\
\hline $\mathrm{N}$ & 199 & 25 & & \\
\hline
\end{tabular}

Nota: Escala 1-10. ***: significativo al 1\%; ** significativo al 5\%; * significativo al $10 \%$.

NS: no significativo; MW: resultados del test no paramétrico de Mann-Whitney de igualdad de medias entre dos grupos; T: resultados del test paramétrico t de Student de igualdad de medias entre dos grupos.

Los promedios para los socios y contratados en cuanto al sentimiento de información son similares. Sin embargo, hay una diferencia importante en el sentimiento de escucha de ambos colectivos, siendo la media de las personas contratadas superior al de las personas socias.

Para saber si la variable situación laboral influye en la percepción de sentirse informado y escuchado, se han utilizado, de nuevo, tanto pruebas paramétricas como no paramétricas, llegando a la conclusión de que la situación laboral influye en la percepción del sentimiento de escucha y no en la percepción sobre la información.

Las entrevistas, por su parte, evidencian que no hay unanimidad a la hora de afirmar si ser socio o contratado influye en la percepción de sentirse informado y escuchado. Por un lado, los contratados no pueden participar en las mismas fuentes de información que los socios: "Los contratados no pueden acceder a algunas vías de información como pueden ser la Asamblea General, el Consejo Rector o al Consejo Directivo". Pero por el lado contrario, la información se transmite sin discriminación alguna: "Los contratados también van a los consejillos". Sin embargo, el hecho de que no haya muchos contratados puede llevar a una falta de evidencias en este aspecto.

Finalmente, se presentan los resultados de la variable sede:

Tabla. 5. Promedios de la percepción del sentimiento de información y de escucha de las personas trabajadoras de Arrasate, Eskoriatza y Usurbil y los contrastes de igualdad de medias entre los distintos grupos

\begin{tabular}{l|l|l|l|l|l} 
& Arrasate & Eskoriatza & Usurbil & $\mathrm{K}-\mathrm{W}$ & ANOVA \\
\hline Información & 6,57 & 5,70 & 5,79 & $\mathrm{X}^{2}=13,21^{* * *}$ & $\mathrm{~F}=6,88^{* * * *}$ \\
\hline Escucha & 5,32 & 4,92 & 5,25 & $\mathrm{X}^{2}=1,16 \mathbf{N S}$ & $\begin{array}{l}\mathrm{F}=0,43 \\
\text { NS }\end{array}$ \\
\hline $\mathrm{N}$ & 116 & 64 & 44 & & \\
\hline
\end{tabular}

Nota: Escala 1-10. ***: significativo al 1\%; ** significativo al 5\%; * significativo al $10 \%$. NS: no significativo; KW: resultados del test no paramétrico de Kruskal-Wallis de igualdad de medias entre más de dos grupos; ANOVA: resultados del test paramétrico F de igualdad de medias entre más de dos grupos. 
El promedio del sentimiento de información de las personas trabajadoras de Arrasate es algo superior al de las personas trabajadoras de Eskoriatza y Usurbil. En el caso de la escucha, los promedios son bastante similares. De hecho, al hacer los contrastes para ver si la variable sede influye en la percepción de sentirse informado y escuchado, sólo en el caso de la información las medias son estadísticamente diferentes, esto es, el sentimiento de información es diferente en las distintas sedes.

Para determinar entre qué sedes hay diferencias significativas, se han realizado contrastes de diferencias de medias entre dos grupos con la prueba no paramétrica de Mann-Whitney y la prueba paramétrica t de Student.

Tabla. 6. Contrastes de igualdad de medias para las distintas comparativas entre sedes

\begin{tabular}{|c|c|c|c|c|}
\hline & & $\begin{array}{l}\text { Arrasate } \\
\text { Eskoriatza }\end{array}$ & $\begin{array}{l}\text { Arrasate } \\
\text { Usurbil }\end{array}$ & $\begin{array}{l}\text { Eskoriatza } \\
\text { Usurbil }\end{array}$ \\
\hline \multirow{2}{*}{ Información } & MW & $2592 * * *$ & $1957 * *$ & 1336 NS \\
\hline & $\mathrm{T}$ & $3,46 * * *$ & $2,61 * *$ &,$- 26 \mathbf{N S}$ \\
\hline \multirow{2}{*}{ Escucha } & MW & 3342 NS & 2504 NS & 1319 NS \\
\hline & $\mathrm{T}$ & ,94 NS & $0,15 \mathbf{~ N S}$ &,$- 60 \mathrm{NS}$ \\
\hline
\end{tabular}

Nota: ***: significativo al 1\%; ** significativo al 5\%; * significativo al $10 \%$. NS: no significativo; MW: resultados del test no paramétrico de Mann-Whitney de igualdad de medias entre dos grupos; T: resultados del test paramétrico t de Student de igualdad de medias entre dos grupos.

Los resultados obtenidos indican que hay diferencias significativas en el sentimiento de información entre la sede de Arrasate y las otras dos sedes.

No obstante, en las entrevistas no hay unanimidad a la hora de afirmar si ser trabajador de las distintas sedes de la cooperativa influye en la percepción de sentirse informado y escuchado. En cuanto a la percepción del sentimiento de información, algunos entrevistados señalan que las diferencias son por la distancia física y la centralización de los servicios: "Todos los servicios generales (personal, gerencia, presidencia...) están en la sede central". Otros, sin embargo, señalan que los canales de información son los mismos para todos y, por consiguiente, la información recibida es la misma: "La información importante llega de la misma manera a un sitio que otro". Sin embargo, en cuanto a la percepción del sentimiento de escucha se refiere, algunos entrevistados indican que en dicha percepción puede influir la distancia física existente entre las distintas sedes y la centralización de los servicios en una de ellas. Otros, por el contrario, indican que a todas las personas trabajadoras se les escucha por igual, independientemente de la sede en la que trabajen.

\section{Discusión de los resultados}

Aunque las personas trabajadoras, por lo general, admiten que tienen la percepción de sentirse informadas en cuanto a objetivos, metas, resultados y demás 
información de la cooperativa, tienen la percepción de que son poco escuchadas por sus superiores cuando les trasladan sus preocupaciones y expectativas. A pesar de todo, en la percepción del sentimiento de información y de escucha de las personas trabajadoras de la cooperativa se dan diferencias estadísticas significativas entre las variables ser miembro de órganos, tipo de mano de obra, sede y situación laboral.

En cuanto a la variable ser miembro de órganos, los datos de la investigación indican que ser miembro o no de órganos -Consejo de Dirección, Consejo Rector y Consejo Social- influye en la percepción del sentimiento de información y de escucha. En cuanto al sentimiento de información se refiere, el resultado está en consonancia con las conclusiones de Thompson (2000) y Lingán (2003), quienes indicaron que la jerarquía influye en la percepción del sentimiento de información y que cuanto mayor es el cargo mayor es el sentimiento de información. Es evidente que los miembros de órganos se sienten más informados que las personas trabajadoras no participantes en órganos. Asimismo, los resultados de las entrevistas evidencian que las personas trabajadoras perciben la influencia mencionada. Para argumentar dicha influencia, el discurso más utilizado es que los miembros de los distintos órganos disponen de más información que el resto de las personas trabajadoras.

Los datos obtenidos de la percepción de escucha de los miembros de órganos están en sintonía con las conclusiones extraídas por Truss y su equipo (2006), quienes indicaron claramente que el cargo, el tipo de contrato y el nivel de estudios de las personas trabajadoras influyen en la percepción del sentimiento de escucha. Es especialmente reseñable el caso de los miembros del Consejo Rector, pues aun siendo el órgano de representación y gobierno de la cooperativa, sus miembros muestran la percepción más baja del sentimiento de escucha, en comparación con el resto de miembros. Ese dato, en cierta medida, choca con lo concluido por Truss y su equipo (2006), que señalaron que las personas encargadas de supervisar el trabajo realizado por sus compañeros se sienten más escuchadas. Asimismo, es remarcable el caso de los miembros del Consejo Social: aun siendo un órgano consultivo que representa el colectivo de socios ante las instancias internas de la cooperativa, en la percepción del sentimiento de escucha, en una escala de 1 a 10 , el resultado medio es inferior a 5. Por consiguiente, queremos destacar que estos resultados, a priori, pueden resultar extraños, ya que se podría pensar que el ser miembro de algún órgano de la cooperativa debería influir positivamente en la percepción del sentimiento de escucha. A su vez, es evidente que no hay unanimidad en las entrevistas a la hora de indicar si la variable miembro de órganos influye en la percepción del sentimiento de escucha.

De igual forma, los resultados de la investigación indican que en la percepción del sentimiento de información y escucha influye el ser persona trabajadora de mano de obra directa e indirecta. Además, las entrevistas evidencian que la gran mayoría de los entrevistados perciben dicha influencia. Según los entrevistados, la influencia de la variable tipo de mano de obra en la percepción del sentimiento de información se debe a que las personas trabajadoras de mano de obra indirecta tienen un mayor número de reuniones y una relación estrecha con las fuentes de información. Esta última evidencia parece estar en sintonía con lo anunciado por Chia-Liang, Shan-Jan y Tse-Pingek (2013), es decir, que las personas trabajadoras 
que participan en proyectos importantes se relacionan y charlan más fácilmente con la parte alta del organigrama, es decir, con las fuentes de información. No obstante, la baja formación y los complejos de las personas trabajadoras, la actitud defensiva de los jefes inmediatos, el sentirse inferior al resto de las personas trabajadoras y el no participar lo suficiente en los distintos foros de la cooperativa son, según los entrevistados, las razones por las que la variable tipo de mano de obra influye en el sentimiento de escucha. Este resultado ofrece más evidencias al contexto estudiado por Bretos, et al. (2018), Heras-Saizarbitoria (2014) y Ortega (2011), entre otros.

Los resultados de la investigación indican que la variable sede influye únicamente en la percepción del sentimiento de información. Estos datos están en sintonía con los resultados de Bustamante (2013b), quien concluyó que en la percepción del sentimiento de información influye el que la persona trabajadora sea de una planta o de otra. Sin embargo los resultados cualitativos indican que las personas trabajadoras no perciben la influencia de forma generalizada. Para algunos la variable sede influye en la percepción del sentimiento de información por la distancia física que hay entre las distintas plantas y por la centralización de los servicios en una sola sede (entre otros: personal, administración, presidencia y gerencia). Esta última afirmación está en sintonía con lo indicado por Goldhaber y su equipo (1978). Para otros, la variable sede no influye en la percepción del sentimiento de información ya que los canales de información son los mismos para todos y, en consecuencia, es la misma información la que llega a las distintas sedes.

Por último, cabe destacar que los resultados indican que el ser socio o contratado de la cooperativa influye únicamente en la percepción del sentimiento de escucha. Aunque pueda parecer paradójico, son los contratados los que disponen de una mejor percepción. Este resultado puede parecer extraño, ya que se podría pensar que los socios deberían tener una opinión más favorable, precisamente, porque al disponer de un mayor número de foros de información se les facilita la participación oral y, por consiguiente, eso debería ayudar a tener una mejor percepción del sentimiento de escucha. Aun así, esta diferencia podría deberse a las expectativas no cubiertas de los socios sobre los foros de información, tal y como indican Heras-Saizarbitoria (2014) y Ortega (2011), porque es limitada la participación de las personas trabajadoras en los órganos de la cooperativa. Las diferencias de expectativas entre socios y contratados constituyen un factor mencionado también por Arando et al. (2015). Puede parecer extraño también el hecho de que la variable situación laboral no influya en la percepción del sentimiento de información. Cabría suponer que los socios deberían tener una mejor percepción del sentimiento de información que los contratados porque tienen acceso a un mayor número de vías de información.

Aunque las entrevistas evidencian que la cooperativa se preocupa por mantener informadas a las personas trabajadoras, no ha habido unanimidad a la hora de señalar si la cooperativa se preocupa por conocer las preocupaciones y expectativas de estas mismas personas. Los miembros del Consejo Rector comparten la opinión de que, a menos que no tengan necesidad expresa, los jefes inmediatos y la alta dirección no realizan esfuerzos suficientes para escuchar a las personas trabajadoras. Esto confirma la conclusión de Macnamara (2015) y Varona (2007) de que la escucha es un acto olvidado y descuidado en el mundo laboral. 


\section{Conclusiones, límites de la investigación y futuras líneas de actuación}

Este estudio no solo complementa las contribuciones realizadas en la literatura sobre gestión empresarial y comunicación interna, sino que también contribuye a alimentar un ámbito de conocimiento muy poco investigado hasta la fecha: las sociedades cooperativas y la comunicación interna. Los distintivos de las cooperativas -entre otros, el carácter dual de la figura de trabajadora-propietaria, los valores, el modelo de gestión participativo, la gobernanza democrática y la responsabilidad social corporativa-, han acaparado multitud de trabajos. No obstante, la comunicación interna ha sido analizada únicamente de forma indirecta por autores que han investigado, entre otros, sobre la gobernanza, la participación o la internacionalización (Bretos, Errasti y Marcuello, 2018; Arando, Gago, Jones, Kato, 2015; Ortega y Uriarte, 2015; Heras-Saizarbitoria, 2014; Ortega, 2011; Altuna, 2008). Si consideramos que trabajos recientes indican que en las empresas convencionales no se ha prestado la suficiente atención a la escucha (Macnamara, 2015; Varona, 2007) y que es preocupante que la escucha organizacional sea poco estudiada (Macnamara, 2014), este trabajo contribuye a ampliar horizontes y a suplir, en cierto modo, este vacío en el estudio de las empresas cooperativas. En consecuencia, una de las principales aportaciones de este estudio es su carácter novedoso, pues, como ya ha sido mencionado, coloca el foco de atención en la comunicación interna de las sociedades cooperativas (más concretamente, en la percepción de las personas trabajadoras sobre la información transmitida por la cooperativa y sobre cómo realizan la escucha los directivos y mandos intermedios de la misma). Tal y como se indica en el recién estrenado Foro de Comunicación de MONDRAGON (TU Lankide, 2018), los resultados de este estudio pueden servir tanto a los directivos de las cooperativas como a los responsables de comunicación, para subrayar el rol estratégico de la comunicación en las cooperativas, compartir experiencias, seguir aprendiendo de las prácticas en esta materia y, observando las carencias percibidas, visualizar las futuras líneas de investigación. Igualmente, puede valer para colaborar en la consolidación de la comunicación interna como herramienta estratégica de dirección.

Esta investigación cuenta con limitaciones que deberían de ser tenidas en cuenta a la hora de interpretar los resultados, con el fin de precisar el alcance de las conclusiones y a la vez abrir nuevas vías de investigación. La investigación se realiza de forma transversal con las personas trabajadoras de una única cooperativa del Grupo MONDRAGON. Los resultados logrados en la investigación mostrarán tendencias, pero no podrán generalizarse a otras cooperativas. Aun así, y teniendo en cuenta que los resultados obtenidos pueden servir a los directivos y responsables de comunicación, se estima interesante realizar investigaciones similares en otras cooperativas. Igualmente, se estima interesante conocer por qué los socios tienen una peor percepción del sentimiento de escucha que los contratados.

\section{Referencias Bibliográfícas}

Altuna, L. (2008) La experiencia cooperativa de MONDRAGON, una sintesis general. Eskoriatza: Lanki. 413 p. 
Arando, S. y Arenaza, I. (2018) Inter-Cooperation Mechanisms in Mondragon: Managing the Crisis of Fagor Electrodomésticos. En: Berry, D.; Kato, T. Employee Ownership and Employee Involvement at Work: Case Studies. Emerald Publishing Limited, pp. 7-35. DOI: $10.1108 / \mathrm{S} 0885-333920180000018008$.

Arando, S., et al. (2015) Efficiency in employee-owned enterprises: An econometric case study of Mondragon. ILR Review, $\mathrm{N}^{\mathrm{o}}$ 2, Vol. 68, pp. 398-425. DOI: 10.1177/0019793914564966.

Bartoli, A. (1992) Comunicación y organización. La organización comunicante y la comunicación organizada. Barcelona: Paidós Ibérica. 222p.

Belategi, O. (2006) Barne komunikazioaren tratamendua MCCko kooperatibetan. Suficiencia investigadora. Eskoriatza: Mondragon Unibertsitatea.

Bisquerra, R. (2009) Metodología de la investigación educativa ( $2^{\circ}$ ed.). Madrid: La Muralla. 456 p.

Bretos, I., Errasti, A. y Marcuello, C. (2018) Ownership, governance, and the diffusion of HRM practices in multinational worker cooperatives: case-study evidence from the Mondragon group. Human Resource Management Journal, $\mathrm{N}^{\mathrm{o}}$ 1, Vol. 28, pp. 76-91. DOI: $10.1111 / 1748-8583.12165$.

Bustamante, E. (2013) El clima de comunicación, la motivación y la satisfacción laboral en un proceso de atención primaria en Colombia. Revista de Comunicación y Salud, $\mathrm{N}^{\mathrm{o}} 1$, Vol. 3, pp. 35-49.

Bustamante, E. (2013b) La comunicación interna y la promoción de la salud. Estudio de caso en Madrid Salud. Tesis Doctoral. Barcelona: Universidad Autónoma de Barcelona.

Castro, E. (2010) El estudio de casos como metodología de investigación y su importancia en la dirección y administración de empresas. Revista Nacional de Administración, $\mathrm{N}^{\mathrm{o}}$ 2, Vol. 1, pp. 31-54.

Catalá, M. (2001) Comunicación interna: atención al mensaje y a la participación en la empresa. Proyecto Social: Revista de relaciones laborales, $\mathrm{N}^{\circ}$ 9, pp. 45-54.

Chetty, S. (1996) The case study method for research in small -and medium - sized firms. International small business journal, $\mathrm{N}^{\mathrm{o}}$ 5, pp. 73-85.

Chia-Liang, H., Shan-Jan, K. y Tse-Ping D. (2013) The relationship between team communication, structure and academic R\&D performance: empirical evidence of the national telecommunication program in Taiwan. $R \& D$ Management, $\mathrm{N}^{\mathrm{o}} 2$, Vol. 43, pp. 121-135. DOI: $10.1111 / \mathrm{radm} .12004$.

Clampitt, P. y Downs, C. (1993) Employee perceptions of the relationship between communication and productivity: a field study. Intenational Journal of Business Communication, $\mathrm{N}^{\mathrm{o}}$ 1, Vol. 30, pp. 5-28. DOI: 10.1177/002194369303000101.

Cornella, A. (2002) Infonomia!com. La gestión inteligente de la información en las organizaciones. Bilbao: Ediciones Deusto. 337 p.

D’aprix, R. (1999) La comunicación para el cambio. Cómo conectar el lugar de trabajo con las cambiantes demandas del mercado, Barcelona: Granica. 204 p.

Del Pozo, M. (1995) La influencia de la cultura empresarial de multinacionales extranjeras en el desarrollo de la comunicación interna de sus filiales españolas. Tesis Doctoral. Madrid: Universidad Complutense de Madrid. Disponible en internet: http://eprints.ucm.es/1818/.

Díaz de Salas, S., Mendoza, V.M. y Porras, C. (2011) Una guía para la elaboración de estudios de caso. Razón y palabra, Vol. 75.

Dircom (2002) Expectativa, prácticas y resultados de la comunicación interna en empresas e instituciones españolas, Madrid: Dircom. 103 p. 
Downs, C.W. (1988) Communication Audits. Glenview, Illinois: Scott, Foresman and Company. $230 \mathrm{p}$.

Errasti, A., Bretos, I. y Nunez A. (2017) The vialbility of cooperatives: the fall of the Mondragon cooperative Fagor. Review of Radical Political Enonomics, No 2, Vol. 49, pp. 181-197. DOI: 10.1177/0486613416666533.

Flick, U. (2007) Introducción a la investigación cualitativa ( $2^{\circ}$ ed.). Madrid: Morata. 324 p.

Goldhaber, G.M. et al. (1978) Organizational communication: 1978. Human Communication Research, $\mathrm{N}^{\mathrm{o}}$ 1, Vol. 5, pp. 76-96. DOI: 10.1111/j.14682958.1978.tb00624x.

Gómez, M. (2006) Introducción a la metodología de la investigación científica. Córdoba: Brujas. 190 p.

Gómez Aguilar, M. (2007) La comunicación en las organizaciones para la mejora de la productividad: el uso de los medios como fuente informativa en empresas $e$ instituciones andaluzas. Tesis Doctoral. Málaga: Universidad de Málaga. Disponible en internet: http://www.biblioteca.uma.es/bbldoc/tesisuma/17672697.pdf

Gonzalo, P. (2012) La transcendencia de escuchar. Predicar con el ejemplo gana a la información. Revista de comunicación, Vol. 24, pp. 64-65.

Heras-Saizarbitoria (2014) The ties that bind? Exploring the basic principles of workerowned organizations in practice. Organization, $\mathrm{N}^{\mathrm{o}}$ 5, Vol. 21, pp. 645-665. DOI: $10.1177 / 1350508414537623$.

Holá, J. (2012) Internal communication in the small and medium sized enterprises. $E+M$ Ekonomika a Management, Vol.3, pp. 32-45.

Hunt, G. y Cusella, L. (1983) A field study of listening needs in organizations. Communication Education, Vol. 32, pp. 393-401. DOI: 10.1080/03634528309378560.

Inforpress (2002) III Estudio sobre la comunicación interna en España. Capital Humano: revista para la integración y desarrollo de los recursos humanos, $\mathrm{N}^{\circ} 159$, pp. 73-85.

Lingán, B.J. (2003) Implicancias conceptuales y metodológicas de la comunicación estratégica en la gestión de la imagen corporativa interna de los servicios sanitarios. Caso: El Hospital Vall d'Hebron. Tesis Doctoral. Universitat Autònoma de Barcelona: Barcelona.

Macnamara, J. (2015) The work and "architecture of listening": requisites for ethical organization-public communication. Ethical Space: The International Journal of Communication Ethics, № 2, Vol. 12, pp. 29-37.

Macnamara, J. (2014) Organisational listening: a vital missing element in public communication and the public sphere. Asia Pacific Public Relations Journal, No 1, Vol. 15, pp. 89-108.

Mishra, K.E. (2007) Internal communication: building trust, commitment, and a positive reputation through relationship management with employees. Tesis Doctoral. Chapel Hill: University of North Carolina.

MONDRAGON (2018) Presencia en el mundo. https://www.mondragoncorporation.com/sobre-nosotros/presencia-en-el-mundo/).

Moreno, I., Martín, C. y Pet, C. (2012) El arte de comunicar en las organizaciones sociales. Málaga: Shepa. $178 \mathrm{p}$.

Nikolic, M., et al. (2012) The impact of internal communication on strategic and economic effects in Serbian companies. Public Relations Review, No 2, Vol. 38, pp. 288-293. DOI: 10.1016/j.pubrev.2011.12.014.

Opitz, I. y Hinner, M.B. (2003) Good internal communication increases productivity. Technical University Bergakademie Freiberg, Faculty of Economics and Business 
Administration. Freiberg working papers, 2003, 07, TU Bergakademie Freiberg, Faculty of Economics and Business. Disponible en: https://tinyurl.com/y 7 fgqfos.

Ortega, I. (2011) Diagnóstico sobre la participación institucional en las cooperativas. Documento sin publicar.

Ortega, I. y Uriarte, L. (2015) Retos y dilemas del cooperativismo de MONDRAGON tras la crisis de Fagor Electrodomésticos. Eskoriatza: Huhezi.

Pérez Serrano, G. (1998) Investigación cualitativa. Retos e interrogantes, II. Técnicas y análisis de datos. Madrid: La Muralla. 234 p.

Rosenbluth, H.F., McFerrin, D. (2004) El cliente es lo segundo. Ponga a sus empleados en primer lugar. Barcelona: Deusto. $247 \mathrm{p}$.

Ruck, K. y Welch, M. (2012) Valuing internal communication; management and employee perspectives, Public Relations Review, Vol. 38, pp. 294-302. DOI: 10.1016/j.pubrev.2011.12.016.

Simard, M., Marchand, A. y Brossard, M. (1990) Les contremaitres et la prévention des accidents du travail en contexte de participation des travailleurs. Québec: Bibliothèque nationale de Québec. Disponible en: https://tinyurl.com/ycbrr6s8.

Smidts, A., Pruyn, H. y Van Riel, C. (2001) The impact of employee communication and perceived external prestige on organizational identification. The Academy of Management Journal, No 5, Vol. 44, pp. 1051-1062. DOI: 10.2307/3069448.

Stake, R.E. (1999) Investigación con estudio de casos. Madrid: Morata. 159 p.

Steil, L.K. y Bommelje, R.K. (2004) Listening leaders. Then ten golden rules to listen, lead and succeed. Ohms Lane: Beaver's Pond Press. 380 p.

Teplow, L. (1956) Communication as a way of industrial life, Management Review, $\mathrm{N}^{\circ} 45$, pp. 702-708.

Tessi, M. (2012) Comunicación interna en la práctica. Siete premisas para la comunicación en el trabajo. Buenos Aires: Granica. 263 p.

Thompson, T.E. (2000) Differences in perception regarding the process of internal communication among administrators, faculty, and staff at a comprehensive southern university. Tesis Doctoral. The University of Southern Mississippi, Hattiesburg.

Timmerman, F. (2000) Cómo ganar el desafío de la información. Bilbao: Deusto. 191 p.

Truss, C., et al. (2006) Working life: employee attitudes and engagement 2006. Research report. Great Bitain: Chartered Institute of Personnel and Development. 54 p.

TU Lankide (2018) La primera edición del Foro de Comunicación cumple sus objetivos. TU Lankide, $\mathrm{N}^{\mathrm{o}} 604$, p. 8.

TU Lankide (2014) MONDRAGON ocupa el primer puesto en el ranking mundial de cooperativas de industria. TU Lankide, $\mathrm{N}^{\circ} 589$, pp. 38-39.

Varona, F. (2007) Multicultural management. La comunicación en la era de la globalización y su impacto en la empresa. Oleiros: Netbiblo. $131 \mathrm{p}$.

Vilanova, N. (2013) Micropoderes. Comunicación interna para empresas con futuro. Barcelona: Plataforma. 137 p.

Villarreal, O. y Landeta, J. (2010) El estudio de casos como metodología de investigación científica en dirección y economía de la empresa. Una aplicación a la internacionalización. Investigaciones Europeas de Dirección y Economía de la Empresa, № 3, Vol.16, pp. 31-52. DOI: 10.1016/S1135-2523(12)60033-1.

Yin, R.K. (2009) Case study research: design and methods, $\mathrm{N}^{\circ}$ 5. Thousand Oaks, CA: Sage Publications. 219 p.

Zelko, H. (1954) An outline of the rol of listening in communications. Journal of Communication, № 3, Vol. 4, pp. 71-75. DOI: 10.1111/j.1460-2466.1954.tb00231.x. 
Zerfass, A. et al. (2015). European Communication Monitor 2015. Creating communication value through listening, messaging and measurement. Results of a survey in 41 countries. Brussels: EACD/EUPRERA, Helios Media. 